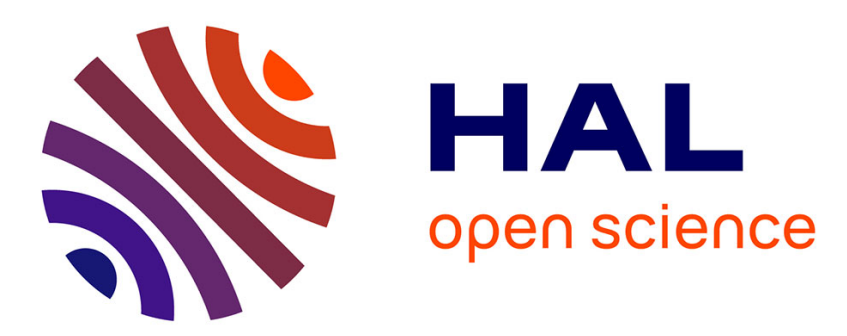

\title{
Volume Dependence in Handel's Model of Quartz Crystal Resonator Noise
}

Fabrice Sthal, Michel Devel, Santunu Ghosh, Joël Imbaud, Gilles Cibiel, Roger Bourquin

\section{- To cite this version:}

Fabrice Sthal, Michel Devel, Santunu Ghosh, Joël Imbaud, Gilles Cibiel, et al.. Volume Dependence in Handel's Model of Quartz Crystal Resonator Noise. IEEE Transactions on Ultrasonics, Ferroelectrics and Frequency Control, 2013, 60, pp.1971 - 1977. 10.1109/TUFFC.2013.2782 · hal-00877311

\section{HAL Id: hal-00877311 \\ https://hal.science/hal-00877311}

Submitted on 28 Oct 2013

HAL is a multi-disciplinary open access archive for the deposit and dissemination of scientific research documents, whether they are published or not. The documents may come from teaching and research institutions in France or abroad, or from public or private research centers.
L'archive ouverte pluridisciplinaire HAL, est destinée au dépôt et à la diffusion de documents scientifiques de niveau recherche, publiés ou non, émanant des établissements d'enseignement et de recherche français ou étrangers, des laboratoires publics ou privés. 
Volume dependence in Handel's model of quartz crystal resonator noise

F. Sthal*, M. Devel*, S. Ghosh*, J. Imbaud*, G. Cibiel ${ }^{\#}$ and R. Bourquin* *FEMTO-ST Institute, UFC, CNRS, ENSMM, UTBM, Besançon, France

${ }^{\#}$ Centre National d’Etudes Spatiales (CNES), Toulouse, France

Email: fsthal@ens2m.fr 


\begin{abstract}
Though criticized by many, Handel's quantum model for $1 / f$ noise remains the only model giving a quantitative estimation of the level of intrinsic $1 / f$ noise in quartz crystal resonators, compatible with the best experimental results. In this paper, we reconsider the volume dependence in this model. We first argue that an acoustic volume, representing the volume in which the vibration energy is trapped, should be used instead of the geometrical volume between the electrodes. Then, we show that since there is an implicit dependence of the quality factor of the resonator with its thickness, the net effect of Handel's formula is not an increase of noise proportionally to the thickness of the resonator, as could be naively expected, but a net decrease when thickness increases. Finally, we show that a plot of $Q^{4} S_{y}$ versus the acoustic volume, instead of the usual $S_{y}$ plot, could be useful to compare the quality of acoustic resonators having very different resonance frequencies.
\end{abstract}




\section{INTRODUCTION}

So far, ultra-stable oscillators (USO) using quartz crystal resonators remain the best solution to have the highest short-term stability in volumes of a few $\mathrm{cm}^{3}$ which is very important for local time references in space applications (for example) since size and weight are important parameters. With low noise electronics at the state of the art, the fundamental limitation for this short-term stability is flicker frequency noise in the resonator (hereafter simply called $1 / f$ noise). Until recently, the best short-term stability for a quartz crystal resonator was measured in 1994 by J. Norton, for a 5 MHz, SC cut, resonator, mounted in an electrode-less structure (BVA) [1]. The corresponding result in terms of Allan standard deviation was 3.74·10 $10^{-14}$ @ $\tau=10 \mathrm{~s}$.

Furthermore, two 10 MHz BVA, SC-cut quartz crystal oscillators were measured in 1999, by R. J. Besson, to have a 5·10 $0^{-14} @ \tau \approx 10 \mathrm{~s}$ Allan standard deviation [2]. This seemed to be the ultimate limit that one could obtain for a quartz based oscillator in the short-term stability domain since, no more tangible solution had been found to improve the phase noise of the resonator itself. Recently, better experimental results [3-5] have reintroduced hopes that quartz crystal oscillators may still have a progression margin in the short-term stability domain and refueled the discussion about what is the main factor limiting the stability between the phase flicker noise in the electronics and the intrinsic noise of the quartz crystal.

One problem is that, even after more than thirty years of research, the detailed physical origin of this intrinsic resonator $1 / f$ noise is still misunderstood. Hence, no entirely satisfactory quantitative predictive model can be given in order to optimize the industrial processes or simply understand the spread of characteristics in a single fabrication batch. Two models have indeed attempted to give quantitative predictions of the level of $1 / f$ noise in quartz resonators, the $1 / f$ quantum noise theory of P. Handel [6] and the degenerated thermal boson theory of M. Planat [7], but the prediction of M. Planat was orders of magnitude above 
the best measurements, maybe because of the use of the traditional phononic density of states despite the fact that all the harmonics of the fundamental modes had already been taken into account in the calculation of the partition function per mode [8].

In 1975, Peter Handel proposed a model to explain the $1 / f$ part of the frequency noise in some electronic systems, using the discrete, random and instantaneous character of soft photon emission by a positively or negatively accelerated charge (bremsstrahlung) [9-12]. In that model, the $1 / f$ noise would be due to the quantum beat in the interferences between the elastic and inelastic scattered waves which emerge when a beam of particles scatters under the influence of a localized perturbation. This perturbation would cause losses of energy quanta of the incident beam, one at a time, each loss being independent from the others. This mechanism has been named "quantum $1 / f$ noise”. Actually, it predicts $1 / f$ noise in any system whenever the interaction rate of the system corresponding to the signal, with its environment, exhibits an infrared divergence due to the generation of low-frequency excitations in quantities increasing when $f$ decreases. It has been applied to several physically different systems by P. Handel (see e.g. [6], [13-19]), among which quartz crystal resonators. In the case of Bulk Acoustic Wave (BAW) quartz resonators at their turn-over temperature ( $\sim 350 \mathrm{~K})$, the fluctuations are mostly caused by the three-phonons process corresponding to the interaction of a phonon from the main resonator mode with a thermal phonon. The result is another thermal phonon with a slightly different frequency and the loss of a phonon in the main resonator mode. Connecting this discrete variation of the number of phonons in some average thermal mode to a discrete variation of the quartz polarization, P. Handel obtained the following formula for the power spectral density of relative frequency fluctuations [14]:

$$
S_{y}(f)=\frac{\beta \cdot V}{Q^{4} f}
$$

with $Q$ the intrinsic quality factor of the resonator, $V\left(\right.$ in $\mathrm{cm}^{3}$ ) the volume of the resonant part inside the resonator, and $\beta$ a proportionality factor dependent on the physical parameters of 
the material, numerically estimated to be of the order of $1 \mathrm{~cm}^{-3}$ in quartz resonators. We note first that this is supposed to represent the fundamental lower limit of noise spectral density at low frequencies, in the resonator, therefore in the oscillator. Second, we recall that A. van der Ziel was able to recover the same formula [20], except for the detailed expression of $\beta$, using semiclassical arguments. However, we should also note that a careful analysis of this detailed analytical expression of $\beta$ and the way it is demonstrated by Handel calls for more precise definitions of some of the quantities occurring in this expression, so as to have an explicit way to numerically evaluate them more precisely [8].

In formula (1), the volume $V$ has been traditionally approximated by the volume between the electrodes [14], but then, the latest experimental results [5] seem to be below the prediction (considering $\beta=1 \mathrm{~cm}^{-3}$ ). In this paper, we show that a better estimation of the theoretical limit may be extracted from Handel's model, using the acoustic volume of the resonator, defined by the trapping of energy, instead of the volume between the electrodes. Additionally, the presence of the volume $V$ in the numerator of (1), instead of in the denominator as naively expected from experimental facts, will be discussed. 


\section{ACOUSTIC VOLUME ESTIMATION}

The acoustic volume estimation is carried out using Tiersten's model as e. $g$. in [21].

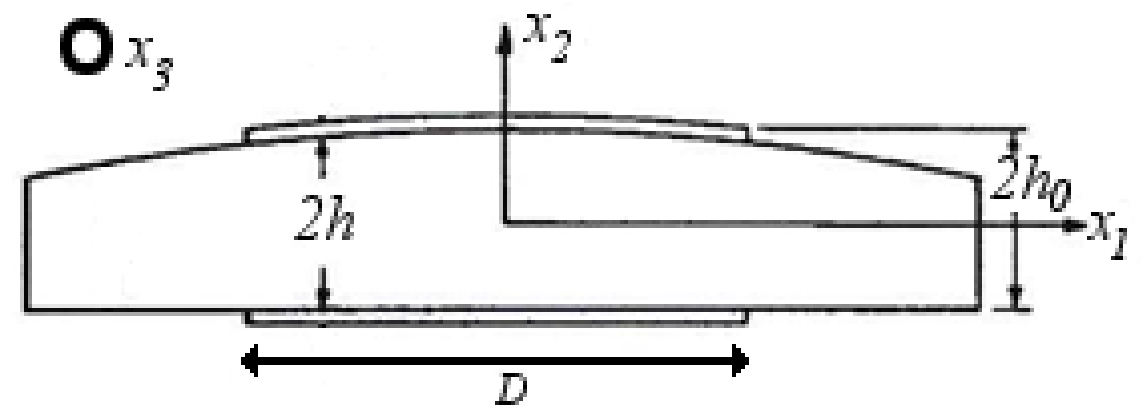

Figure 1. Geomeric definition of a plano-convex resonator

Due to the plano-convex shape of a resonator (Fig. 1), the thickness $2 h$ of the resonator at some off-axis point with coordinates $\left(x_{1}, x_{2}, x_{3}\right)$, is given by the following expression:

$$
2 h \approx 2 h_{0}\left[1-\frac{\left(x_{1}^{2}+x_{3}^{2}\right)}{4 R h_{0}}\right]
$$

where $h_{0}$ is the maximum height of the resonator (on the $x_{2}$ axis) and $R$ the curvature radius of the convex face. Furthermore, for stationary shear waves propagating in the $x_{2}$ direction, the amplitude of the mechanical displacement modes in the resonator can be approximated by [21]:

$u_{n m p} \approx A \sin \left(\frac{n \pi x_{2}}{2 h}\right) H_{m}\left(\sqrt{\alpha_{n}} x_{1}\right) H_{p}\left(\sqrt{\beta_{n}} x_{3}\right) \exp \left(-\alpha_{n} x_{1}^{2} / 2-\beta_{n} x_{3}^{2} / 2\right) \exp \left(j \omega_{m n p} t\right)$

with:

$$
\alpha_{n}^{2}=\frac{n^{2} \pi^{2} \hat{c}}{8 R h_{0}^{3} M^{\prime}{ }_{n}} \text { and } \beta_{n}^{2}=\frac{n^{2} \pi^{2} \hat{c}}{8 R h_{0}^{3} P_{n}^{\prime}}
$$

where $n=1,3,5, \ldots$ is the overtone (OT) number, $m, p=0,2,4, \ldots$, label the different mode shapes in the plane of the resonator, $H_{m}$ and $H_{p}$ are Hermite polynomials, $M^{\prime}{ }_{n}$ and $P^{\prime}{ }_{n}$ are the dispersion constants and $\hat{c}$ is the effective elastic constant associated to the propagation of this kind of mode. 
For the $3^{\text {rd }}$ overtone (OT 3), we consequently have:

$$
u_{300} \approx A \sin \left(\frac{3 \pi x_{2}}{2 h}\right) \exp \left(-\alpha_{3} x_{1}^{2} / 2-\beta_{3} x_{3}^{2} / 2\right) \exp \left(j \omega_{300} t\right)
$$

with

$$
\alpha_{3}^{2}=\frac{9 \pi^{2} \hat{c}}{8 R h_{0}^{3} M_{3}^{\prime}} \text { and } \beta_{3}^{2}=\frac{9 \pi^{2} \hat{c}}{8 R h_{0}^{3} P_{3}^{\prime}}
$$

The eigenfrequency $\omega_{300}$ is obtained from:

$$
\omega_{300}^{2}=\frac{9 \pi^{2} \hat{c}}{4 h_{0}^{2} \rho}\left[1+\frac{1}{3 \pi} \sqrt{\frac{2 h_{0}}{R}}\left(\sqrt{\frac{M_{3}^{\prime}}{\hat{c}}}+\sqrt{\frac{P_{3}^{\prime}}{\hat{c}}}\right)\right]
$$

The elastic energy in the resonator is approximately given by:

$$
W_{1} \approx \frac{A^{2}}{2} \hat{c}\left(\frac{3 \pi}{2 h_{0}}\right)^{2} \iiint_{\mathrm{V}} \cos ^{2}\left(\frac{3 \pi x_{2}}{2 h_{0}}\right) \exp \left(-\alpha_{3} x_{1}^{2}-\beta_{3} x_{3}^{2}\right) d V
$$

We then define the acoustic volume as the volume that would contain the same elastic energy, for a hypothetical wave that would have constant amplitude in the $\left(x_{1}, x_{3}\right)$ plane, for a resonator with two plane electrodes $(1 / R=0)$ of equivalent surface $S_{e q}$ separated by a distance $2 h_{0}$. For this hypothetical resonator, the trapped elastic energy would be:

$$
W_{2} \approx \frac{A^{2}}{2} \hat{c}\left(\frac{3 \pi}{2 h_{0}}\right)^{2} \int_{-h_{0}}^{h_{0}} \cos ^{2}\left(\frac{3 \pi x_{2}}{2 h_{0}}\right) d x_{2} \times S_{e q}
$$

Thus:

$$
W_{1}=W_{2} \Rightarrow V_{a c}=2 h_{0} S_{e q}=2 h_{0} \iint \exp \left(-\alpha_{3} x_{1}^{2}-\beta_{3} x_{3}^{2}\right) d x_{1} d x_{3}
$$

However, thanks to the Gaussian functions, as long as the electrodes diameter $D>4 / \operatorname{Min}\left(\sqrt{\alpha_{3}}, \sqrt{\beta_{3}}\right)$, their real outer shape is not important and the diameter can even be taken to be infinite. Using the fact that $\int_{-\infty}^{\infty} \exp \left(-\alpha x^{2}\right) d x=\sqrt{\pi / \alpha}$, we then find that:

$$
V_{a c}=2 h_{0} S_{e q} \approx 2 h_{0} \pi / \sqrt{\alpha_{3} \beta_{3}}=\sqrt{32 R h_{0}^{5} \sqrt{M_{3}^{\prime} P_{3}^{\prime}} / 9 \hat{c}}
$$




\section{APPLICATION OF THE MODELS TO EXPERIMENTAL MEASUREMENTS}

As seen in [5], the best measured resonators are the $5 \mathrm{MHz}$ BVA SC-cut, $3^{\text {rd }}$ OT Cmode, manufactured by the Oscilloquartz S.A. Company (Swatch Group), based in Neuchatel, Switzerland. Since for SC-cut quartz resonators, the cut is defined by a double rotation $\left(\varphi=22^{\circ} 45^{\prime}, \theta=34^{\circ}\right)$, the relevant effective parameters are $\hat{c} \approx 34.6 \mathrm{GPa}, M_{3}^{\prime} \approx 57 \mathrm{GPa}$ and $P_{3}^{\prime} \approx 67 \mathrm{GPa}$ [22]. In [23], a succinct geometrical description of the resonator is given which allows us to estimate the diameter of the resonant part of the resonator (around $20 \mathrm{~mm}$ ) and the electrodes diameter $D$ (about $11 \mathrm{~mm}$ ). Using (7) and the motional capacitance of the prototype resonator measured in [3], we can estimate the thickness $2 h_{0} \approx 1.097 \mathrm{~mm}$ and the radius of curvature of the electrodes $R \approx 146 \mathrm{~mm}$ ( $c f$. appendix). This leads to $1 / \sqrt{\alpha_{3}} \approx 1.38 \mathrm{~mm}$ and $1 / \sqrt{\beta_{3}} \approx 1.43 \mathrm{~mm}$. Using (11), we can now compute $V_{a c}=6.81 \cdot 10^{-9} \mathrm{~m}^{3}$, whereas a direct numerical integration of (10) in polar coordinates, gives $V_{a c}=6.80 \cdot 10^{-9} \mathrm{~m}^{3}$.

One can then verify that the applicability condition $D>4 / \operatorname{Min}\left(\sqrt{\alpha_{3}}, \sqrt{\beta_{3}}\right)$ stated in the previous paragraph is indeed numerically verified in this case. As argued in the previous paragraph, in contoured resonators, the energy trapping is quasi-independent of the electrodes dimension, provided they are big enough, though they influence the motional elements and the static capacitor of the Butterworth - van Dyke equivalent circuit. Indeed, for such a radius of curvature the energy density at the edge of the volume between the electrodes is at least $2 \cdot 10^{6}$ smaller than the energy density at the same height on the axis.

In order to use Handel's formula (1), we still need to compute the quality factor $Q$ of this kind of resonator, which can be obtained using the following equation:

$$
Q=\frac{\hat{c}}{2 \pi f \hat{\eta}}
$$


In our case, $\widehat{\eta} \approx 3.95 \cdot 10^{-4} \mathrm{Ns} / \mathrm{m}^{2}$ [24], which turns into an estimation of the maximum quality factor of about $2.79 \cdot 10^{6}$. This value corresponds well with the one given in [3] $\left(2.7 \cdot 10^{6}\right)$

We now turn to the experimental quantities that we could compare to Handel's $1 / f$ noise threshold. The short-term stability is usually given in the time domain by the Allan variance [25], which is the variance of the difference of the average fractional frequencies measured for two consecutive samples of time length $\tau$. It can be computed in the frequency domain by using the power spectral density of frequency fluctuations [26]:

$$
\sigma_{y}^{2}(\tau)=\int_{0}^{\infty} S_{y}(f) \frac{2 \sin ^{4}(\pi f \tau)}{(\pi f \tau)^{2}} d f
$$

In the case of flicker frequency noise (which is the limiting resonator noise at low frequencies), the Allan standard deviation corresponding to the power spectral density (PSD) of relative frequency fluctuations $S_{y}(f)=S_{y}(1 \mathrm{~Hz}) / f$ turns out to be independent of $\tau$ and constitutes the floor of the noise at low frequencies. It is the given by the expression [26]:

$$
\sigma_{y_{-} \text {flicker }}=\sqrt{2 \ln (2) S_{y}(1 H z)}
$$

Table 1 presents the comparison between measurements and the results of Handel's model, considering both definitions of volume in (1) (and $\beta=1 \mathrm{~cm}^{-3}$ ). One can see that the limit set by Handel's model with $\beta=1 \mathrm{~cm}^{-3}$, is indeed below the experimental results if one uses the acoustic volume defined in this paper, whereas it is not if one uses the geometrical volume between electrodes. 


\begin{tabular}{|c|c|}
\hline Frequency (MHz) & 5 \\
\hline Quality factor $Q\left(10^{6}\right)$ & 2.79 \\
\hline Volume under electrodes $\mathrm{V}_{\mathrm{el}}\left(\mathrm{cm}^{3}\right)$ & $1.043 \cdot 10^{-1}$ \\
\hline Acoustic volume $\mathrm{V}_{\mathrm{ac}}\left(\mathrm{cm}^{3}\right)$ & $0.681 \cdot 10^{-2}$ \\
\hline Volume ratio & 15 \\
\hline $\begin{array}{l}\sigma_{\mathrm{y}_{\text {_floor_Handel }}} \\
\text { (vol. under electrodes) }\end{array}$ & $4.89 \cdot 10^{-14}$ \\
\hline $\begin{array}{l}\sigma_{y_{-} \text {floor_Handel }} \\
\text { (Acoustic vol.) }\end{array}$ & $1.25 \cdot 10^{-14}$ \\
\hline $\begin{array}{l}\sigma_{y_{-} \text {floor_exp }[1]} \\
\text { (oscillator measurement) }\end{array}$ & $3.75 \cdot 10^{-14}$ \\
\hline $\begin{array}{l}\sigma_{y_{f} \text { floor_exp [4] }} \\
\text { (oscillator measurement) }\end{array}$ & $2.5 \cdot 10^{-14}$ \\
\hline
\end{tabular}

\section{SIZE DEPENDENCE}

A point that can be a priori surprising with Handel's formula is the proportionality of $S_{y}(1 \mathrm{~Hz})$ with the volume. On Fig. 2, we plotted experimental values for $S_{y}(1 \mathrm{~Hz})$ as a function of the volume $V_{e l}$ between the electrodes, using results from various publications [12], [27-28], including those used by Handel to justify his model and recent results obtained for $5 \mathrm{MHz}$ oscillators specially designed by FEMTO-ST for industrials partners. One can easily see that a linear increase of $S_{y}(1 \mathrm{~Hz})$ with $V_{e l}$ is NOT supported by the experimental results. This seems at first sight to be a very serious problem for Handel's theory, but if we look closer at (1), we see that there are other factors in this equation that may also depend on the geometrical dimensions of the resonator. 


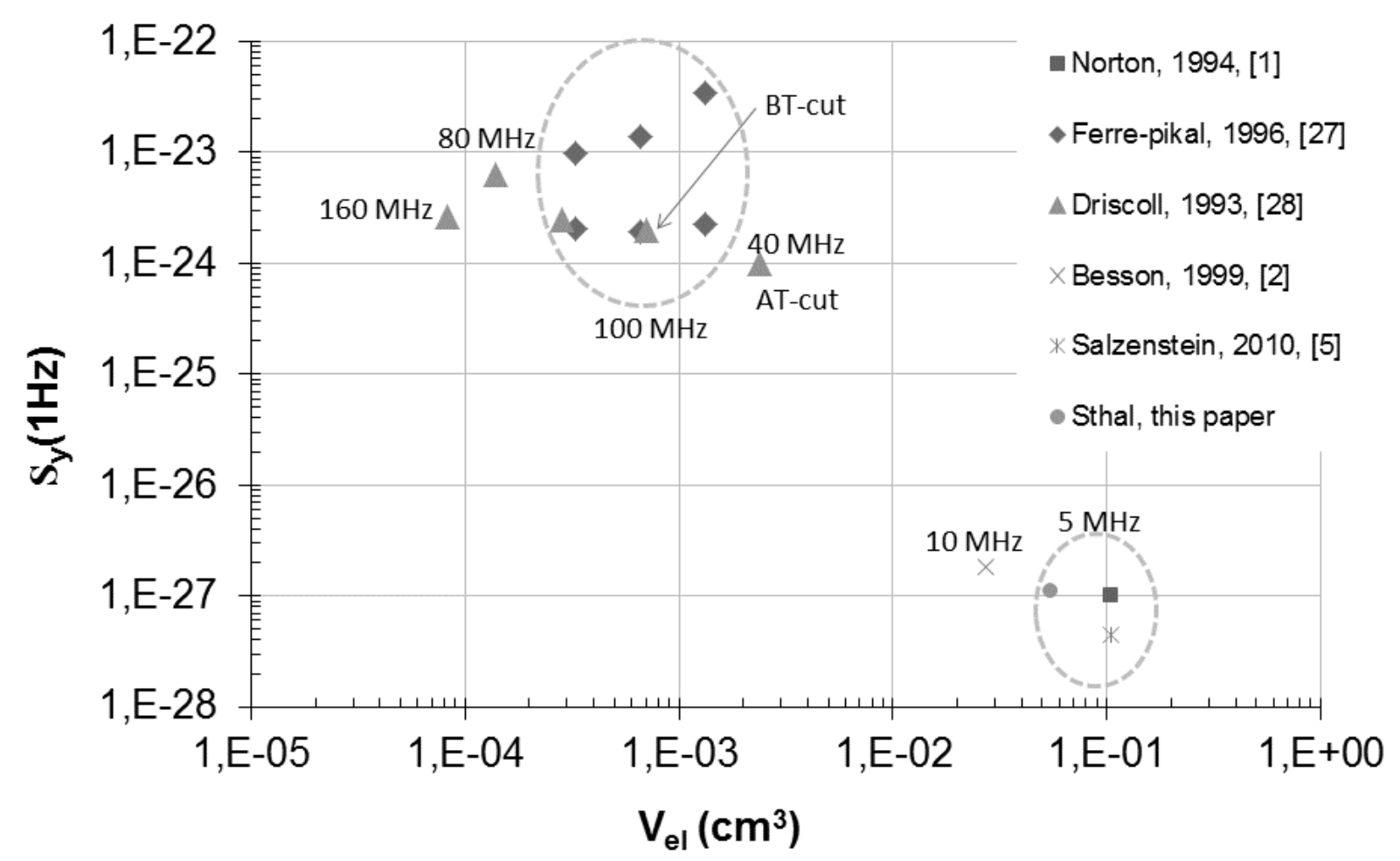

Figure 2. $\quad S_{y}(1 \mathrm{~Hz})$ as a function of the volume between the electrodes of the resonator for experimental points from various authors.

Indeed, a plot of the $Q^{4} S_{y}(1 \mathrm{~Hz})$ product for the same experiments, as a function of $V_{e l}$ (Fig. 3), gives a very different trend: the volume dependence is now similar to that predicted by Handel in his model! This is due to the fact that $Q^{4}$ also depends on the dimensions of the resonator (particularly its thickness $2 h_{0}$ ). This introduces a hidden dependence with the size of the resonator that we will study in more details below. However, several points seem to have a lower noise than the intrinsic limit supposedly set by Handel's model if one uses $\beta=1 \mathrm{~cm}^{-3}$ as proposed in Handel's papers. At this point, one could either question this $\beta=1 \mathrm{~cm}^{-3}$ value, as we did in another paper [8], or use the above-defined acoustic volume, describing the volume in which elastic energy is confined, instead of the geometric volume between the electrodes. 


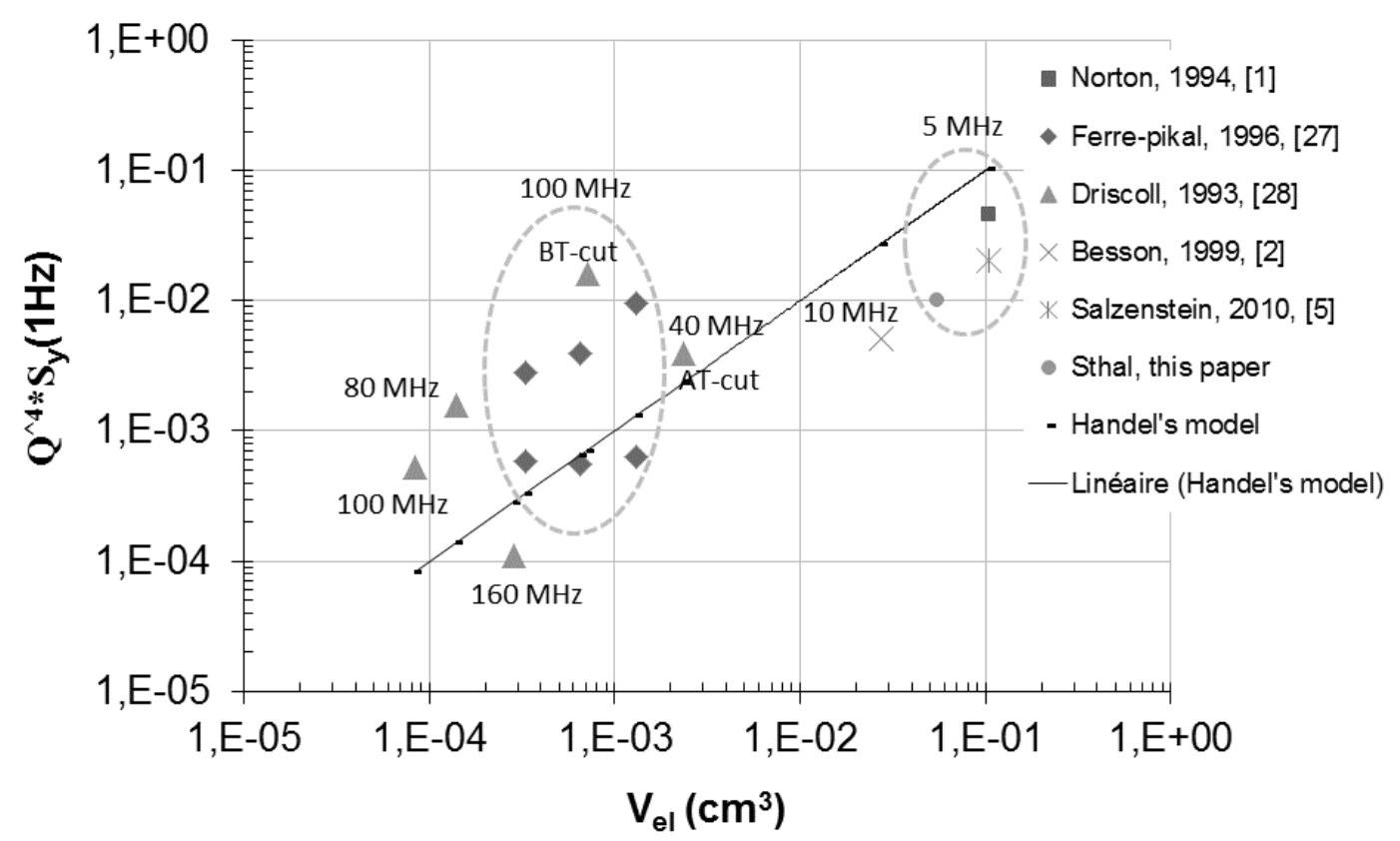

Figure 3. $\quad Q^{4} \cdot S_{y}$ as a function of the volume between the electrodes of the resonator, experimental points from various authors + straight line for Handel's prediction with

$$
\beta=1 \mathrm{~cm}^{-3} \text {. }
$$

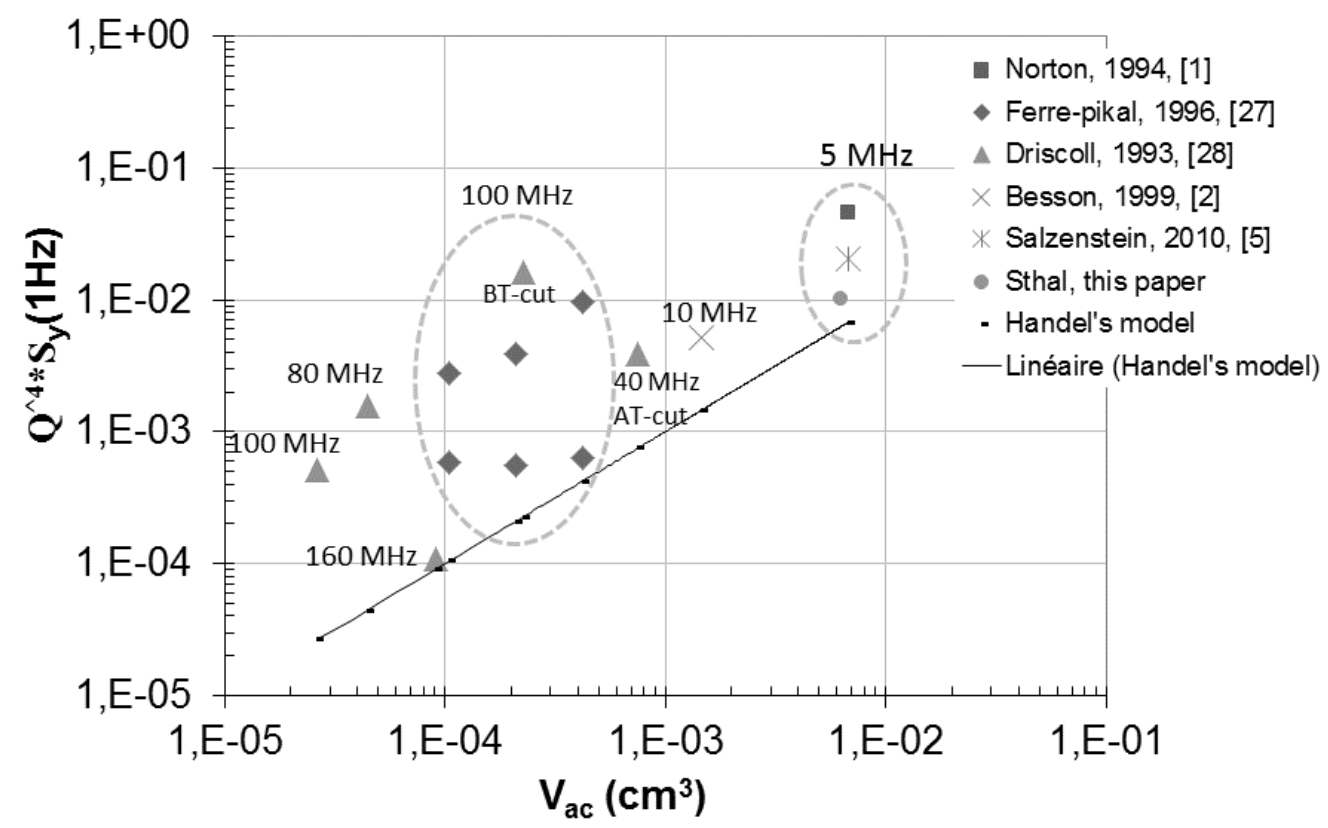

Figure 4. $\quad Q^{4} \cdot S_{y}$ as a function of the acoustic volume of the resonator (given by (11) for plano-convex resonators and (17) for plano-plano resonators), experimental points from various authors + straight line for Handel's prediction with $\beta=1 \mathrm{~cm}^{-3}$. 
For plano-convex resonators, formula (10) was used instead of the volume between electrodes to draw Fig. 4. However, higher frequency resonators have a plano-plano shape and we had to use another formula for the acoustic volume. According to [21] section III, the trapped energy mode shape can then be approximated by products of simple trigonometric functions in such resonators, the trapped energy $W_{1}$ is computed from the following equation:

$$
W_{1} \approx \frac{A^{2}}{2} \hat{c}\left(\frac{n \pi}{2 h_{0}}\right)^{2} \iiint_{\mathrm{V}}\left[\cos \left(\frac{n \pi x_{2}}{2 h_{0}}\right) \cos \left(\frac{\pi x_{1}}{2 l}\right) \cos \left(\frac{\pi x_{3}}{2 l}\right)\right]^{2} d V
$$

with $l$ the half width of a square electrode. At this point, following Stevens and Tiersten, we consider that circular electrodes can be approximated by square electrodes with a width equal to the radius of the real electrodes. Thus using (9), we find:

$$
\begin{gathered}
W_{1}=W_{2} \Rightarrow V_{a c}=2 h_{0} S_{e q}=2 h_{0} \int_{-l}^{l} \int_{-l}^{l}\left[\cos \left(\frac{\pi x_{1}}{2 l}\right) \cos \left(\frac{\pi x_{3}}{2 l}\right)\right]^{2} d x_{1} d x_{3} \\
V_{a c}=2 h_{0} S_{e q} \approx 2 h_{0} l^{2} \approx V_{e l} / 4
\end{gathered}
$$

In the plano-plano case, the acoustic volume $V_{a c}$ is simply proportional to the thickness of the resonator and to the area of the electrodes and equals a fourth of the volume between the electrodes. Data from [27-28], necessary to compute $V_{a c}$ for these high frequency plano-plano resonators, are recalled in table 2 . We note that we only kept the best measurements from [27], in order to draw Fig. 4.

TABLE II. RESONATOR PARAMETERS FROM [27-28] USED TO DRAW FIG. 4. Q FACTOR OF RESONATORS FROM [27] HAVE BEEN FOUND MORE PRECISELY IN [29].

\begin{tabular}{|l|c|c|c|c|c|c|}
\hline Reference & {$[27]$} & {$[27]$} & {$[27]$} & {$[28]$} & {$[28]$} & {$[28]$} \\
\hline frequency $(\mathrm{MHz})$ & 100 & 100 & 100 & 80 & 100 & 160 \\
\hline Overtone & 5 & 5 & 5 & 3 & 3 & 5 \\
\hline Crystal-Cut & $\mathrm{SC}$ & $\mathrm{SC}$ & $\mathrm{SC}$ & $\mathrm{SC}$ & $\mathrm{SC}$ & $\mathrm{SC}$ \\
\hline Unloaded quality factor & $1.3 \cdot 10^{5}$ & $1.3 \cdot 10^{5}$ & $1.3 \cdot 10^{5}$ & $1.25 \cdot 10^{5}$ & $1.19 \cdot 10^{5}$ & $1.25 \cdot 10^{5}$ \\
\hline Electrode diameter $(\mathrm{mm})$ & 2.16 & 3.05 & 4.32 & 1.63 & 1.40 & 2.54 \\
\hline Resonator Thickness $(\mu \mathrm{m})$ & 90 & 90 & 90 & 67.8 & 54.4 & 56.7 \\
\hline
\end{tabular}


Looking at Fig. 4, one can see that the line corresponding to Handel's model is now lower than all the experimental points. Furthermore, the proportionality of $Q^{4} S_{y}$ with $V_{a c}$ for the best resonators now seems reasonable. Finally this kind of $Q^{4} S_{y}$ plot seems to be useful to compare the performance of resonators with various resonant frequencies.

Since the comparison of the previous graphs clearly shows that one should not forget that the quality factor depends on the physical dimensions of the resonator, we study in more details the thickness dependence of $V / Q^{4}$. For that purpose, we recall that, in the expression (12) of the intrinsic quality factor of an acoustic resonator, $f \sim 1 / h_{0}$, hence $Q \sim h_{0}$. Given the $h_{0}$ dependence of $Q$ and $V$, we then get for constant area electrodes, an inverse proportionality of $S_{y}$ with $h_{0}^{3}$ if $V$ is the volume between the electrodes, or $S_{y} \sim 1 / h_{0}^{3 / 2}$, if $V$ is the acoustic volume. In both cases, noise reduction should be enhanced by an increase of thickness. In high purity crystals, this trend seems to be confirmed by the fact that $5 \mathrm{MHz}$ resonators (2h $1 \mathrm{~mm}$ ) have so far shown short-term stabilities below that of $10 \mathrm{MHz}$ resonators $(2 h \sim 0,5$ $\mathrm{mm})$.

\section{CONCLUSION}

From the previous considerations, we showed how the intrinsic lower limit for $1 / f$ noise limiting short term stability, set by Handel's model can be reconciled with the best latest experimental results, provided one uses the acoustic volume computed from Tiersten's model, instead of the volume between the electrodes. We also showed how Handel's model can be qualitatively very useful in order to compare the quality of oscillators of various resonant frequencies, thanks to the $Q^{4} \cdot S_{y}$ plot. Furthermore, we showed how Handel's model is not in contradiction with the fact that usually thicker resonators exhibit less noise, despite an apparent proportionality of the noise with the volume of the resonator in this model. 


\section{Acknowledgment}

The authors would like to thank P. H. Handel for giving them time and a helpful discussion, and the Centre National d'Etudes Spatiales for the project's financial support.

\section{APPENDIX : ESTIMATION OF THE THICKNESS AND CURVATURE RADIUS}

Using Stevens and Tiersten's results [21], we can relate the motional capacitance $C_{m o t}$ of the resonator, for the $3^{\text {rd }} \mathrm{OT}$, to the thickness and curvature radius of the resonator:

$$
C_{m o t}=\frac{64 \hat{e}_{26}^{2}}{9 \pi^{3} h_{0} \hat{c}} \sqrt{\alpha_{3} \beta_{3}}\left[\int_{0}^{D / 2} \exp \left(-\alpha_{3} x_{1}^{2} / 2\right) d x_{1} \int_{0}^{D / 2} \exp \left(-\beta_{3} x_{3}^{2} / 2\right) d x_{3}\right]^{2}
$$

with $D$ the electrodes diameter and $\hat{e}_{26}=-0.0576 \mathrm{C} / \mathrm{m}^{2}$ the effective piezoelectric constant.

Since, for the same specific kind of resonator that was used for the record measurement [5], the motional capacitance was measured to be $0.195 \mathrm{fF}$ in [3], we have together with (7) of the main text, a set of two nonlinear equations in $h_{0}$ and $R$. In order to solve them numerically, we compute initial estimates of $h_{0}$ and $R$ using first the fact that the thickness at the center of the resonator $2 h_{0}$ is approximately given by $2 h_{0} \approx 3 \lambda / 2$. In the present case, since the acoustic velocity for the $5 \mathrm{MHz}$ thickness shear C-mode in a SC-cut quartz crystal is $\sqrt{\hat{c} / \rho} \approx 3613 \mathrm{~m} / \mathrm{s}\left(\rho=2650 \mathrm{~kg} / \mathrm{m}^{3}\right)$, we find that $2 h_{0} \approx 1.084 \mathrm{~mm}$. Then, we use the fact that the electrodes diameter is always chosen big enough so that the two Gaussian integrals in (A.1) can be approximated by $\sqrt{\pi / 2 \alpha_{3}}$ and $\sqrt{\pi / 2 \beta_{3}}$ respectively. Thus,

$$
C_{m o t} \approx \frac{16 \hat{e}_{26}^{2}}{9 \pi h_{0} \hat{c} \sqrt{\alpha_{3 \beta_{3}}}}=\frac{32 \hat{e}_{26}^{2}}{\pi^{2}(9 \hat{c})^{3 / 2}} \sqrt{2 h_{0} R \sqrt{M_{3}^{\prime} P_{3}^{\prime}}}
$$

Accordingly, the initial value for $R$, can be estimated by:

$$
R=\frac{1}{2 h_{0} \sqrt{M_{3}^{\prime} P_{3}^{\prime}}}\left(\frac{\pi^{2}(9 \hat{c})^{3 / 2} C_{m o t}}{32 \hat{e}_{26}^{2}}\right)^{2}
$$


With $C_{m o t} \approx 0.195 \mathrm{fF}$, we find $R \approx 148 \mathrm{~mm}$. Then, by solving together (7) and (A.1) (with $D=11 \mathrm{~mm}, \quad c f$. main text), we refine our estimation to $2 h_{0} \approx 1.097 \mathrm{~mm}$ and $R \approx 146,6 \pm 0,2 \mathrm{~mm}$ (depending whether the integrations are performed for a square in Cartesian coordinates or for a disk in polar coordinates).

Finally, we note, that if we would know the static capacitance $C_{0}$ of the same resonator, we could estimate $D$ by adding the following formula [21]:

$$
C_{0}=\frac{\pi D^{2}}{4}\left(1+\frac{(D / 2)^{2}}{8 R h_{0}}\right) \frac{\varepsilon_{22}+\hat{e}_{26}^{2} / \hat{c}}{2 h_{0}}
$$

to the set of nonlinear equations to solve. Conversely, using $\varepsilon_{22}=39.78 \cdot 10^{-12} \mathrm{~F} / \mathrm{m}$ the permittivity constant corresponding to the $x_{2}$ direction in the SC-cut and $D=11 \mathrm{~mm}$, we estimate $C_{0} \approx 3.6 \mathrm{pF}$. 


\section{References}

[1] J. R. Norton, "Performance of ultrastable quartz oscillators using BVA resonators,” Proc. European Frequency and Time Forum, Weihenstephan, Germany, March 1994, pp. 457465.

[2] R. J. Besson, M. Mourey, S. Galliou, F. Marionnet, F. Gonzalez, P. Guillemot, R. Tjoelker, W. Diener, A. Kirk, "10 MHz Hyperstable quartz oscillators,” Proc. Joint Meeting IEEE Ann. Freq. Cont. Symp. and European Frequency and Time Forum, Besançon, France, april 1999, pp. 326-330.

[3] J. Chauvin, P. Weber, J.P. Aubry, F. Lefebvre, F. Sthal, S. Galliou, E. Rubiola and X. Vacheret, "A new generation of very high stability BVA oscillators,” Proc. Joint Meeting IEEE Ann. Freq. Cont. Symp. and European Frequency and Time Forum, Genova, Switzerland, June 2007, pp. 1261-1268.

[4] A. Kuna, J. Cermak, L. Sojdr, P. Salzenstein and F. Lefebvre, "Lowest flicker-frequency floor measured on BVA Oscillators,” IEEE Trans. Ultrason., Ferroelec., Freq. Contr., vol. 56, no. 3, pp. 548-551, 2009.

[5] P. Salzenstein, A. Kuna, L. Sojdr and J. Chauvin, "Significant step in ultra high stability quartz crystal oscillators,” Electron. Lett., vol. 46, no. 21, pp. 1433-1434, 2010.

[6] P. H. Handel and A. G. Tournier, "Nanoscale engineering for reducing phase noise in electronic devices,” Proc. IEEE, vol. 93, no. 10, pp. 1784-1814, 2005.

[7] M. Planat, "Thermal $1 / f$ noise from the theory of partitions: application to a quartz resonator," Physica A, vol. 318, pp. 371-386, 2003.

[8] M. Devel, R. Bourquin, S. Ghosh, J. Imbaud, G. Cibiel and F. Sthal, "Quartz crystal resonator noise and fluctuation-dissipation theorem considerations,” Proc. IEEE Int. Freq. Cont. Symp., Baltimore, USA, 22-24 May, pp.425-429, 2012 
[9] P. H. Handel, “1/f noise - an infrared phenomenom,” Phys. Rev. Lett., vol. 34, no. 24, pp. 1492-1495, 1975.

[10] P. H. Handel, "Nature of 1/f phase noise,” Phys. Rev. Lett., vol. 34, no. 24, pp. 14951498, 1975.

[11] P. H. Handel, “Quantum theory of 1/f noise,” Physics Letters, vol. 53A, no. 6, pp. 438440, 1975.

[12] P. H. Handel, "1/f macroscopic quantum fluctuations of electric currents due to bremsstrahlung with infrared radiative corrections,” Zeitschrift fur Naturforschung A, vol. 30, pp. 1201-1202, 1975.

[13] P. H. Handel, “1/f noise universality in high-technology applications,” in Proc. IEEE Int. Freq. Contr. Symp., Boston, Massachusetts, 1994, pp. 8-21.

[14] F. L. Walls, P. H. Handel, R. Besson, and J. J. Gagnepain, “A new model of $1 / f$ noise in BAW quartz resonators,” in IEEE Int. Freq. Contr. Symp., Hershey, Pennsylvania, 1992, pp. 327-333.

[15] P. H. Handel, A. Tournier, and B. Henning, "Quantum 1/f effect in resonant biochemical piezoelectric and MEMS sensors,” IEEE Trans. Ultrason., Ferroelec., Freq. Contr., vol. 52, no. 9, pp. 1461-1467, 2005.

[16] P. H. Handel, "Incoherence and negative entropy in the quantum $1 / f$ effect of BAW and SAW quartz resonators,” in IEEE Int. Freq. Contr. Symp., Orlando, 1997, pp. 464-469.

[17] P. H. Handel, "Quantum 1/f quartz resonator theory versus experiment," in Joint Meeting EFTF - IEEE Int. Freq. Contr. Symp., Besancon, France, 1999, pp. 1192-1195.

[18] J. J. Gagnepain, J. Uebersfeld, G. Goujon, P. Handel, "Relation between 1/f noise and Q-factor in quartz resonators,” Proc. IEEE Ann. Freq. Cont. Symp., Ft. Monmouth, New Jersey, May 1981, pp. 476-483. 
[19] P. H. Handel, "Nature of $1 / f$ frequency fluctuations in quartz resonators," Solid State Electron., vol. 22, pp. 875-876, 1979.

[20] A. van der Ziel, Semiclassical derivation of Handel's expression for the Hooge parameter," J. Appl. Phys.vol. 63, no. 7, pp. 2456-2457, 1988.

[21] D. S. Stevens, H. F. Tiersten, "An analysis of doubly rotated quartz resonators utilizing essentially thickness modes with transverse variation," J. Acous. Soc. Am., vol. 79, $n^{\circ}$ 6, pp. 1811-1826, 1986.

[22] R. Bourquin, B. Dulmet, J. J. Boy, "SC-cut resonator operating in anharmonic modes with B-mode reduction," Proc. European Frequency and Time Forum, Brighton, United Kingdom, March 1996, pp. 239-243.

[23] D. Schneuwly, Y. Schwab, "Quartz clocks," $3^{\text {rd }}$ Int. Telec. Sync. Forum, London, UK, Oct. 2005.

[24] J. Lamb, J. Richter, "Anisotropic acoustic attenuation with new measurements for quartz at room temperatures", Proc., Roy. Soc. Series A, vol. 293, pp. 479-492, 1966.

[25] D. W. Allan,"Statistics of atomic frequency standards,” Proc. IEEE, vol. 54, no. 2, Feb., pp. 136-154, 1966.

[26] IEEE, "IEEE Standard Definitions of Physical Quantities for Fundamental Frequency and Time Metrology - Random Instabilities," in IEEE STD 1139-2008: IEEE, 2008, pp. 1-35.

[27] E.S. Ferre-Pikal, F. L. Walls, J. R. Vig, J. F. Garcia Nava, "Experimental studies on Flicker noise in quartz crystal resonators as a function of electrode volume, drive current, type of quartz, and fabrication process," Proc. IEEE Ann. Freq. Cont. Symp., Honolulu, Hawaii, June 1996, pp. 844-851. 
[28] M. M. Driscoll, W. P. Hanson, "Measured vs. volume model-predicted flicker-offrequency instability in VHF quartz crystal resonators," Proc. IEEE Ann. Freq. Cont. Symp., Salt Lake City, Utah, June 1993, pp. 186-192.

[29] F. L. Walls, "The quest to understand and reduce 1/f noise in amplifiers and BAW quartz oscillators,” Proc. IEEE European Time and Freq. Forum, Besançon, France, 8-10 March, 1995, pp. 227-243. 